\title{
Sudden and Persistent Bradycardia: An Unexpected Indicator of Pin-Site Extradural Hematoma in a Pediatric Patient
}

\author{
Roneeta Nandi ${ }^{1}$ Shruti Redhu ${ }^{1}$ Rana Patir ${ }^{2}$ Hari Hara Dash ${ }^{1}$ \\ ${ }^{1}$ Department of Anesthesiology and Pain, Fortis Memorial Research \\ Institute, Gurugram, Haryana, India \\ 2Department of Neurosurgery, Fortis Memorial Research Institute, \\ Gurugram, Haryana, India

\begin{abstract}
Address for correspondence Roneeta Nandi, DNB, Department of Anesthesiology and Pain, Fortis Memorial Research Institute, Sec. 44, Gurugram 122002, Haryana, India
\end{abstract} \\ (e-mail: delphinium80@gmail.com).
}
Abstract
Keywords
- bradycardia
- cranial fixation
- depressed skull fracture
- extradural hematoma
- pediatric
- pin-site complications
- posterior fossa

Depressed skull fracture and extradural hematoma are infrequent complications of skull pin fixation in children undergoing craniotomy. Neurosurgeons are often alerted about these complications by intraoperative brain swelling or postoperative neurological deterioration. We describe the development of pin-site extradural hematoma in a child on whom Mayfield skull clamp was applied during posterior fossa tumor excision. Sudden and persistent bradycardia observed by the anesthesiologist served as the sole warning sign. Such an on-table indicator of pin-site extradural hematoma has not been described earlier. The anesthesiologist must maintain vigilance and effective communication with surgical colleagues to ensure early detection and timely management of these pin-site complications.

\section{Introduction}

Mayfield skull clamp (Cincinnati, Ohio, United States) is routinely used during neurosurgeries. A plethora of complications following this method of three-point cranial fixation has been reported. Pin-site depressed skull fracture and extradural hematoma are infrequent complications associated with the use of this device. ${ }^{1-7}$ In most cases, intraoperative brain swelling ${ }^{2-4}$ or postoperative neurological deterioration ${ }^{5-7}$ alerts the neurosurgeon to investigate further for these complications.

We report a case of depressed skull fracture and extradural hematoma following posterior fossa tumor excision in a child on whom Mayfield skull clamp was applied. Sudden bradycardia at the end of surgery was the only clinical sign that prompted the anesthesiologist to insist on computed tomographic (CT) scan of the head for further investigation. Such a predictor for this pin-site complication in a child undergoing posterior fossa craniotomy has not been reported earlier. Etiopathogenesis of sudden occurrence of bradycardia has also been discussed.
Written informed consent was obtained from the parent of the patient prior to writing this report.

\section{Case Report}

A 4-year-old boy, weighing $25 \mathrm{~kg}$, with history of intermittent headache for 6 months and gait imbalance for 1 month, was diagnosed with a posterior fossa mass. His contrast-enhanced magnetic resonance imaging (MRI) scan of the brain showed a posterior fossa mass $(5.2 \mathrm{~cm} \times$ $5.1 \mathrm{~cm} \times 4.9 \mathrm{~cm}$ ) with moderate obstructive hydrocephalus with no midline shift (-Fig. 1). A midline suboccipital craniotomy for tumor decompression was planned. Clinical examination of the child did not reveal any abnormality. His biochemical and hematological investigations were within normal limits.

Anesthesia was induced with sevoflurane $8 \%$ in a nitrous oxide and oxygen mixture (50:50). Intravenous (IV) access was secured. Propofol $50 \mathrm{mg}$ was administered. Fentanyl $40 \mu \mathrm{g}$ was used for analgesia. Rocuronium $25 \mathrm{mg}$ was
DOI https://doi.org/ $10.1055 / \mathrm{s}-0038-1660691$ ISSN 2348-0548.
Copyright $\odot 2018$ Indian Society of Neuroanaesthesiology and Critical Care
License terms

$($ (1) $\Theta \circledast$ 


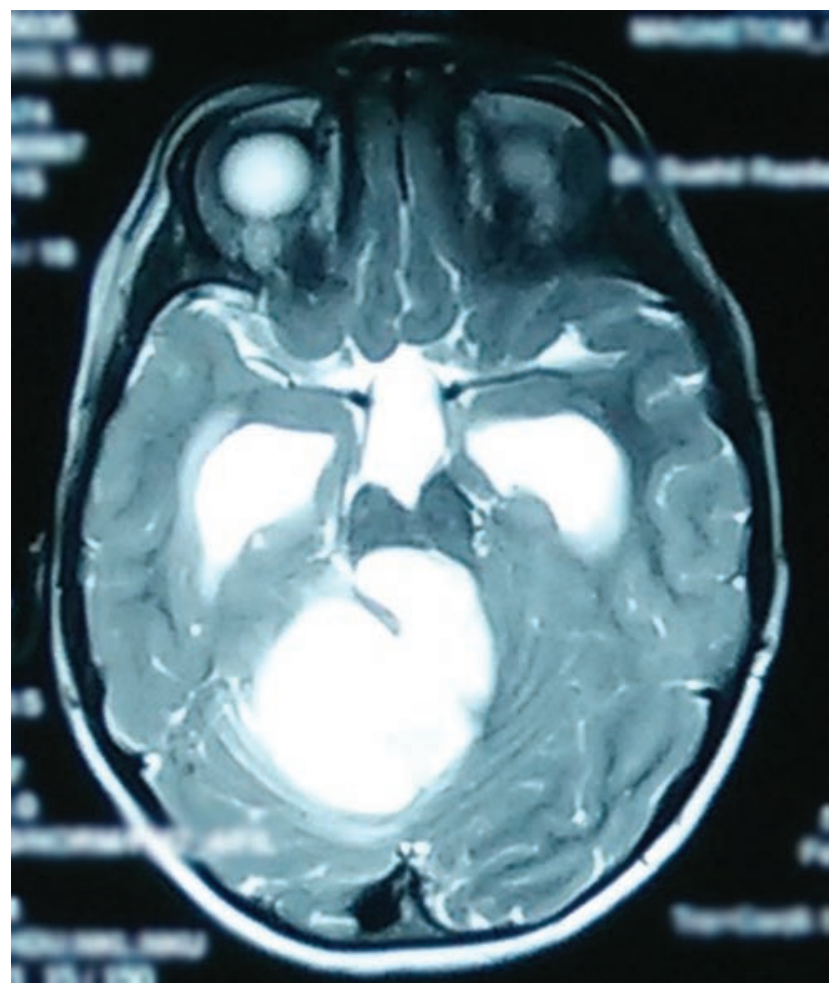

Fig. 1 Contrast-enhanced MRI scan of brain showing posterior fossa mass $(5.2 \mathrm{~cm} \times 5.1 \mathrm{~cm} \times 4.9 \mathrm{~cm})$ with moderate obstructive hydrocephalus with no midline shift. MRI, magnetic resonance imaging.

administered to facilitate orotracheal intubation with a $5.5-\mathrm{mm}$ internal diameter cuffed oral endotracheal tube. Left radial artery was cannulated. Continuous monitoring of electrocardiogram (ECG), $\mathrm{SaO}_{2}$, intra-arterial blood pressure, end-tidal $\mathrm{CO}_{2}\left(\mathrm{ETCO}_{2}\right)$, core temperature, and urine output was performed. Anesthesia was maintained with sevoflurane in air and oxygen (50:50), fentanyl, rocuronium, and intermittent positive-pressure ventilation. $\mathrm{ETCO}_{2}$ was maintained around $30 \mathrm{~mm} \mathrm{Hg}$.

The child was secured in the prone position with his head placed in a three-point skull fixation device (Mayfield) using pediatric pins tightened to $40 \mathrm{lb}$. A single pin on the right parietal bone and a pin each on left temporal and left occipital bones were applied. Near-total excision of the tumor was accomplished in 7 hours. The patient remained hemodynamically stable and normothermic throughout the surgery. Blood loss was estimated to be approximately $600 \mathrm{~mL}$. The child received $1,500 \mathrm{~mL}$ of Ringer's lactate and $1,500 \mathrm{~mL}$ of normal saline, as well as $250 \mathrm{~mL}$ of packed cells. Urine output was $1,000 \mathrm{~mL}$. Intraoperative arterial blood gas (ABG) ( $\mathrm{pH} 7.4 / \mathrm{PO}_{2}$ 197.6/ $\mathrm{PCO}_{2} 36.2 / \mathrm{HCO}_{3}{ }^{-} 22.4$ l base deficit -1.2) showed no abnormalities. At the end of surgery, to remove the Mayfield headrest, the right parietal cranial pin was first unfastened. A spurt of blood was noted at this site. It was partially controlled with pressure, and the remaining two pins along with the Mayfield head rest were removed. The patient was immediately turned supine, and the pin-site bleeding was finally stopped with a suture. The patient seemed to be awakening normally from anesthesia with no apparent neurological deficits. Prior to reversal of residual neuromuscular blockade, the patient's heart rate suddenly dropped from 102 to 58 beats/min, accompanied by a systolic blood pressure of 120 to $130 \mathrm{~mm} \mathrm{Hg}$. Anticholinergic drugs were not administered as blood pressure remained stable. Pupils were normal and reacting to light. In view of persistent bradycardia, the anesthesiologist urged the neurosurgeon to undertake an emergency CT scan of the head. The CT revealed an evolving large right parietal extradural hematoma with an overlying depressed fracture of the right parietal bone (-Fig. 2). There was approximately $15 \mathrm{~mm}$ midline shift to the left side. The patient immediately underwent right

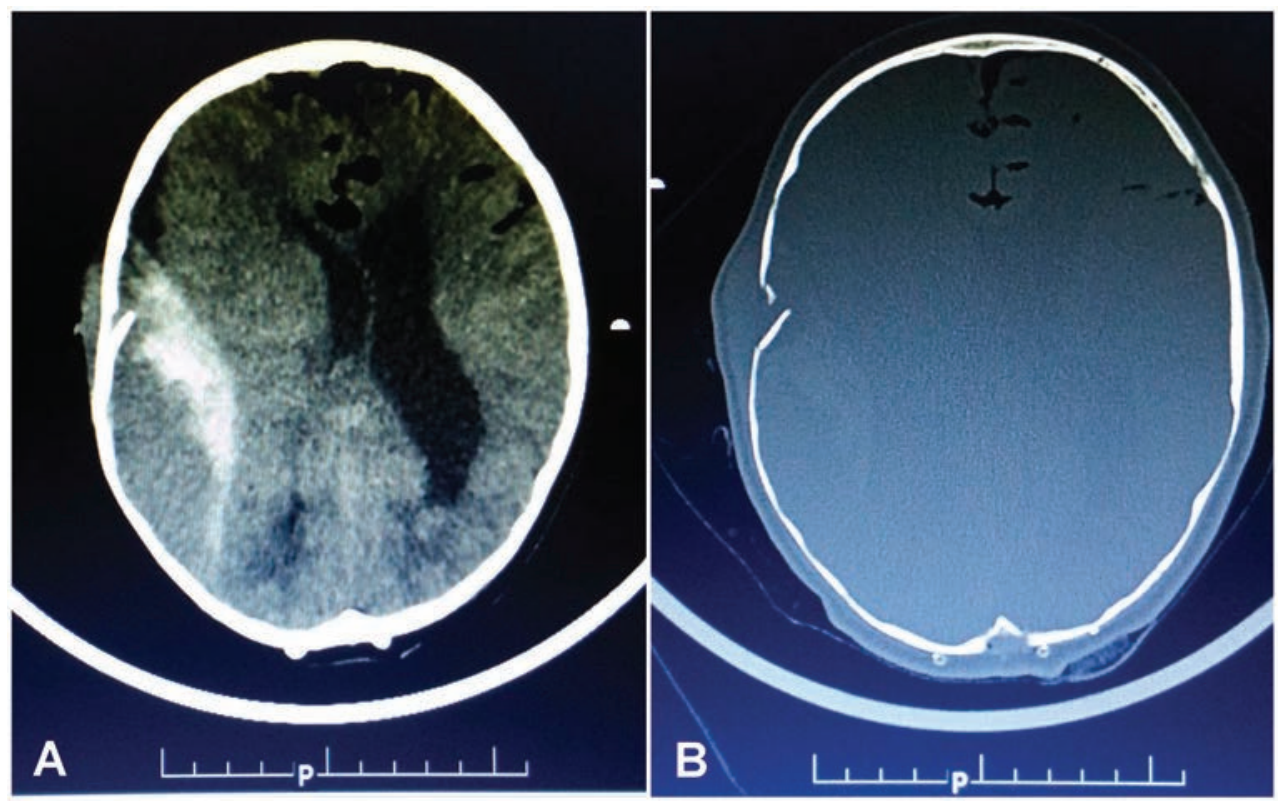

Fig. 2 (A) Postoperative emergent noncontrast computed tomographic scan of the head revealed an evolving large right parietal extradural hematoma, with (B) underlying depressed fracture of the right parietal bone (where the pin had been placed). 
temporoparietal craniotomy for evacuation of the extradural hematoma. Anesthetic induction was done with propofol $30 \mathrm{mg}$, fentanyl $20 \mu \mathrm{g}$, and rocuronium $5 \mathrm{mg}$. Sevoflurane 2 to 3\% in oxygen and air was used for maintenance. Intermittent positive-pressure ventilation maintained $\mathrm{ETCO}_{2}$ in the range of 30 to $35 \mathrm{~mm} \mathrm{Hg}$. Blood loss was approximately $200 \mathrm{~mL}$. Crystalloids $(1,000 \mathrm{~mL})$ and packed cells $(100 \mathrm{~mL})$ were transfused. Urine output was approximately $500 \mathrm{~mL}$ during the 2-hour procedure. Levetiracetam $500 \mathrm{mg}$ was administered for seizure prophylaxis. Bradycardia persisted until craniotomy was achieved approximately 40 minutes after the initial drop in heart rate. Thereafter, heart rate stabilized at 100 to 110 beats/ min. The child was electively ventilated till the first postoperative day and extubated on second postoperative day with no neurological deficit. He was discharged from the hospital in a stable neurological state 1 week after the surgery.

\section{Discussion}

Acute extradural hematoma following head-pin fixation is an infrequent but serious complication following neurosurgery. ${ }^{1-7}$ Early detection and timely management play a vital for ensuring complete functional recovery of the patient.

In this case, a sudden spurt of blood from the right parietal pin site alerted us to the possibility of vascular injury or intracranial hematoma. However, it was the sudden and persistent bradycardia that led us to further investigation. Lo and behold, a depressed fracture of the right parietal bone and a large evolving right parietal extradural hematoma was revealed. This case is unique because it highlights sudden and persistent bradycardia as the sole, on-table indicator of an evolving extradural hematoma in a child on whom Mayfield three-point skull clamp was used. In previous reports of pin-site extradural hematoma, either acute intraoperative brain swelling, ${ }^{2-4}$ postoperative neurological deterioration, or onset of new neurological deficits ${ }^{5-7}$ has led to the diagnosis.

Deciphering the etiopathogenesis of the bradycardia in this case was intriguing. Bradycardia may have been due to (1) Cushing's reflex as a result of intracranial hypertension caused by increasing mass effect of the extradural hematoma or (2) an unusual manifestation of the trigeminocardiac reflex (TCR) following stretch and compression of the dura by the expanding hematoma volume. Sensory innervation of supratentorial dura mater is mainly by meningeal branches of the trigeminal nerve. Stimulation of this nerve anywhere along its course may cause reflex bradycardia for as long as the stimulation persists. ${ }^{8}$ By most definitions of TCR, the bradycardia is accompanied by sudden hypotension. This was not observed in our patient. Instead, the blood pressure remained stable and slightly in the higher range. More significantly, once the intracranial hypertension was relieved by craniotomy, the heart rate normalized. Therefore, the most plausible explanation for the bradycardia observed in our patient is Cushing's reflex.

Depressed skull fracture and extradural hematoma are potentially fatal complications of skull pin fixation in children undergoing craniotomy. Isolated, sudden, and persistent bradycardia at the end of posterior fossa surgery may serve as an immediate on-table warning sign. It is imperative that the anesthesiologist maintains vigilance and effective communication with surgical colleagues.

\section{Funding}

None.

\section{Conflict of Interest}

None.

\section{References}

1 Baerts WD, de Lange JJ, Booij LHDJ, Broere G. Complications of the Mayfield skull clamp. Anesthesiology 1984;61(4):460-461

2 Chen TF, Yang SH, Tsai JC. Depressed skull fracture and epidural hematoma resulted from pin-type head holder for craniotomy in children. J Med Sci 2014;34:238-240

3 Krishnan P, Kumar SK, Kartikueyan R, Patel SM. Pin-site epidural hematoma: A catastrophic complication of cranial fixation in a child. J Neurosci Rural Pract 2016;7(2):286-289

4 Naik V, Goyal N, Agrawal D. Pin site bilateral epidural hematoma - a rare complication of using Mayfield clamp in neurosurgery. Neurol India 2011;59(4):649-651

5 Lee M, Rezai AR, Chou J. Depressed skull fractures in children secondary to skull clamp fixation devices. Pediatr Neurosurg 1994;21(3):174-177, discussion 178

6 Vitali AM, Steinbok P. Depressed skull fracture and epidural hematoma from head fixation with pins for craniotomy in children. Childs Nerv Syst 2008;24(8):917-923, discussion 925

7 Sade B, Mohr G. Depressed skull fracture and epidural haematoma: an unusual post-operative complication of pin headrest in an adult. Acta Neurochir (Wien) 2005;147(1):101-103

8 Abdulazim A, Steinen MN, Sadr-Eshkevari P, et al. Trigeminocardiac reflex in neurosurgery - current knowledge and prospects. In: Signorelli F. Explicative Cases of Controversial Issues in Neurosurgery. London, UK: InTech Open; 2012. Available at: https://www.intechopen.com/books/ explicative-cases-of-controversial-issues-in-neurosurgery/ the-trigeminocardiac-reflex-in-neurosurgery-current-knowledge-and-prospects. Accessed February 6, 2018. 